\title{
Measuring the educational benefits of using a virtual patient to practice pharmacist-patient consultations
}

\author{
Charlotte Lucy Richardson ${ }^{1}$ (iD) , Stephen Chapman², Simon White ${ }^{2}$ iD \\ 1 School of Pharmacy, Faculty of Medical Sciences, Newcastle University, United Kingdom \\ 2 School of Pharmacy and Bioengineering, Keele University, United Kingdom
}

Keywords
Counselling
Education
Pharmacist
Simulation
Virtual patient
Correspondence
Charlotte Lucy Richardson
School of Pharmacy
Faculty of Medical Sciences
Newcastle University
Newcastle NE1 7RU
United Kingdom
charlotte.richardson2@newcastle.ac.uk

\begin{abstract}
Objective: Virtual patients (VPs) are a method of simulating clinical practice however little is known about their use by healthcare professionals. This study explores if, and how, one VP is educationally beneficial for pharmacists and pre-registration trainees to teach non-vitamin $\mathrm{K}$ oral anticoagulant (NOAC) patient counselling. Method: The evaluation used a before and after questionnaire measuring self-perceived counselling ability; further questions considered the outcomes of VP use. Data were analysed using descriptive and inferential statistics; ethical approval was granted by Keele University. Results: There was an average improvement in the user's $(n=94)$ self-perceived ability to conduct NOAC counselling $(+10.2 \%)$. Although, there was variation in educational outcomes with changes in both knowledge and confidence reported. Some reported a decreased ability, but they were still positive towards the VP as it may have had a regulatory effect. Conclusion: Most users perceived multiple benefits of use; more generic outcomes related to counselling skills were also reported.
\end{abstract}

\section{Introduction}

Virtual patients (VPs) are a well-established method of simulating clinical practice with recognised benefits of safety and standardisation (Douglass et al., 2013; Stevens et al., 2006), although they have principally only been assessed for use by undergraduate healthcare students (Al-Dahir et al., 2014; Battaglia et al., 2012; Bindoff et al., 2014; Cavaco \& Madeira, 2012; Menendez et al., 2015; Pereira \& Cavaco, 2014; Smith \& Benedict, 2015; Smith, Mohammad, \& Benedict, 2014) and little is known about the use of VPs outside of a structured educational course and ran by qualified healthcare professionals as opposed to students (Richardson, White, \& Chapman, 2019). VP applications have an established educational value in student groups whereby they have demonstrated uses for knowledge (Battaglia et al., 2012; Zlotos et al., 2016) competency (Bindoff et al., 2014) and performance (Taglieri et al., 2017).
Studies have tended to focus on individual VP technologies built into well-structured undergraduate courses with a single outcome of relevance to a particular application, such as a gain in knowledge or competence; this is in part due to the historic user group being students enrolled on a course rather than registered professionals working in practice. Furthermore, what has not been considered is a fluid and individualised approach to the VP's educational benefits whereby a VP may have benefits around a number of outcomes and types of learning dependent on the individual user and their practice.

No studies have used registered pharmacists as the main user of a VP and so the perspectives of this user group on VP technology and its educational value is unknown; Battaglia and authors (2012) did include pharmacists in their study alongside students but this only accounted for $42(34.4 \%)$ of the participants and determining use in 


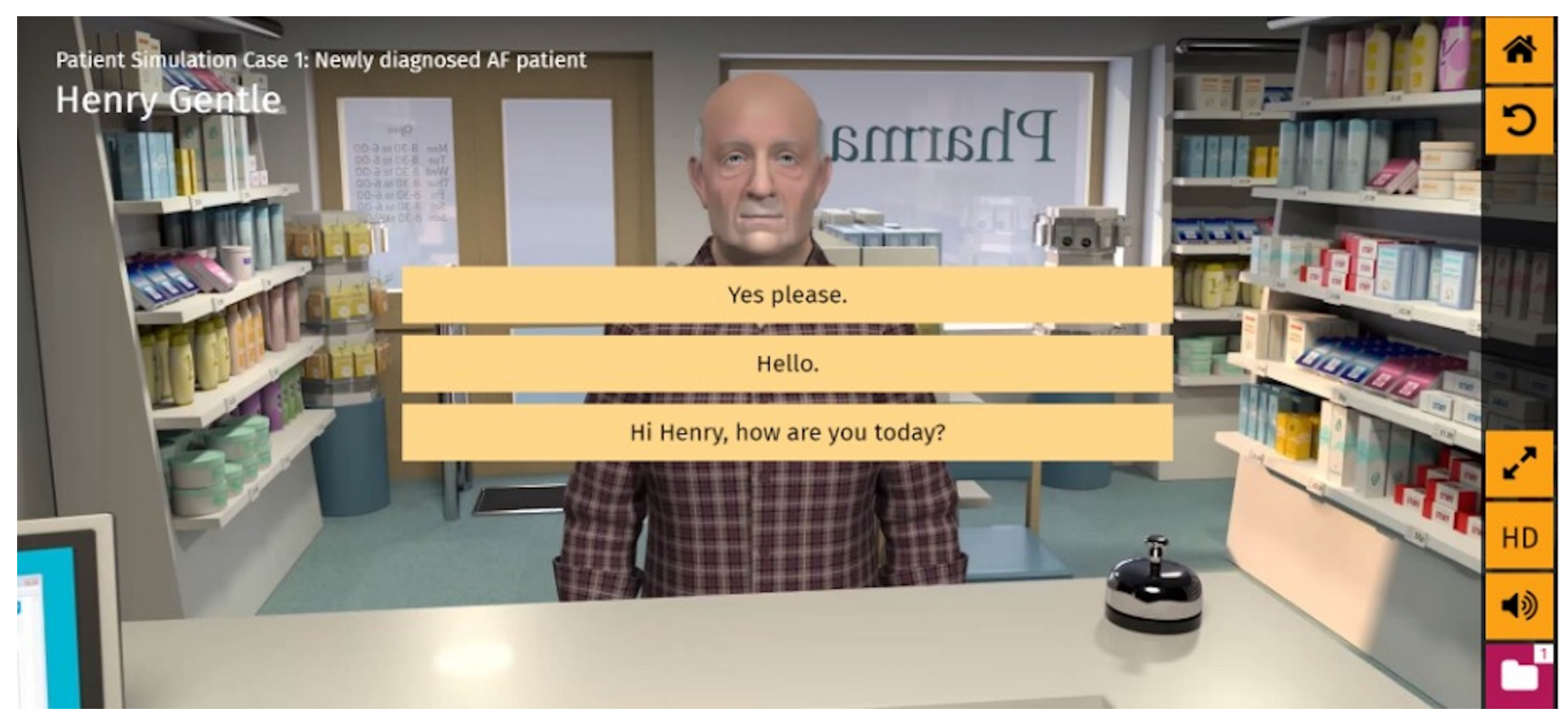

Figure 1: The virtual patient programme interface

pharmacists as the main user group was not an aim of the research; furthermore the technology was static and nonanimated with a focus away from counselling but on medication management (Battaglia et al., 2012). Thompson, White, and Chapman (2017) and Zlotos and authors (2016) both evaluated the use of VPs by pre-registration trainee pharmacists; although in both studies the VP was accessed as part of other education and training as part of the pre-registration training year rather than having a focus on abilities in practice.

In an earlier systematic review, eight VP studies that used animated technology were evaluated and despite all of the applications being aimed at students or preregistration trainees the users, in all cases, took on the role of a pharmacist when using the application. This suggests that VPs are well-suited to simulate a pharmacist's role despite this not yet being directly tested (Richardson, White, \& Chapman, 2019). The possibility to teach qualified HCPs including pharmacists has been highlighted as a future research area by multiple studies (Bracegirdle \& Chapman, 2010; Duff, Miller, \& Bruce, 2016; Kane-Gill \& Smithburger, 2011; Thompson, White, \& Chapman, 2016; Thompson et al., 2017),

Within this study, a VP application has been designed using animated video technology to simulate a patient counselling interaction (Figure 1) (Richardson, Chapman, \& White, 2019). It uses multiple choice questions to give the user choice in the flow and direction of the consultation and it provides personalised feedback to help improve performance and promote reflection. The VP application was developed to teach and provide a practice opportunity for pharmacists and pre-registration trainee pharmacists to develop skills in non-vitamin $\mathrm{K}$ oral anticoagulant (NOAC) patient counselling. This study aims to explore if and how the VP is educationally beneficial for pharmacists and pre-registration trainee pharmacists. Throughout this study there is a focus on the individual learner and personal benefits to using the VP.

\section{Methods}

The VP evaluation used an online questionnaire using Google Forms made up of several elements and question types. Ethical approval from Keele University and United Kingdom Health Research Authority approval were granted prior to data collection; respondents provided informed consent prior to taking part. Respondents were registered pharmacists and pre-registration trainee pharmacists working in hospital and/or community sectors; respondents were recruited using professional networks from 14 sites across England. Recruitment from each used a convenience sample; a sample size calculation was not appropriate for this evaluation as a second part of the evaluation used qualitative methods.

Prior to trialling the VP, respondents were asked a series of Likert questions regarding their current confidence and the likely interest in further NOAC education and training. These questions used 5-point Likert scales of 1 'not at all' to 5 'extremely'. The questionnaire also included before and after tests of the user's self-perceived ability to conduct different elements of NOAC counselling, this was based on the 11 items discussed within the National Institute for Health and Care Excellence guidelines for 


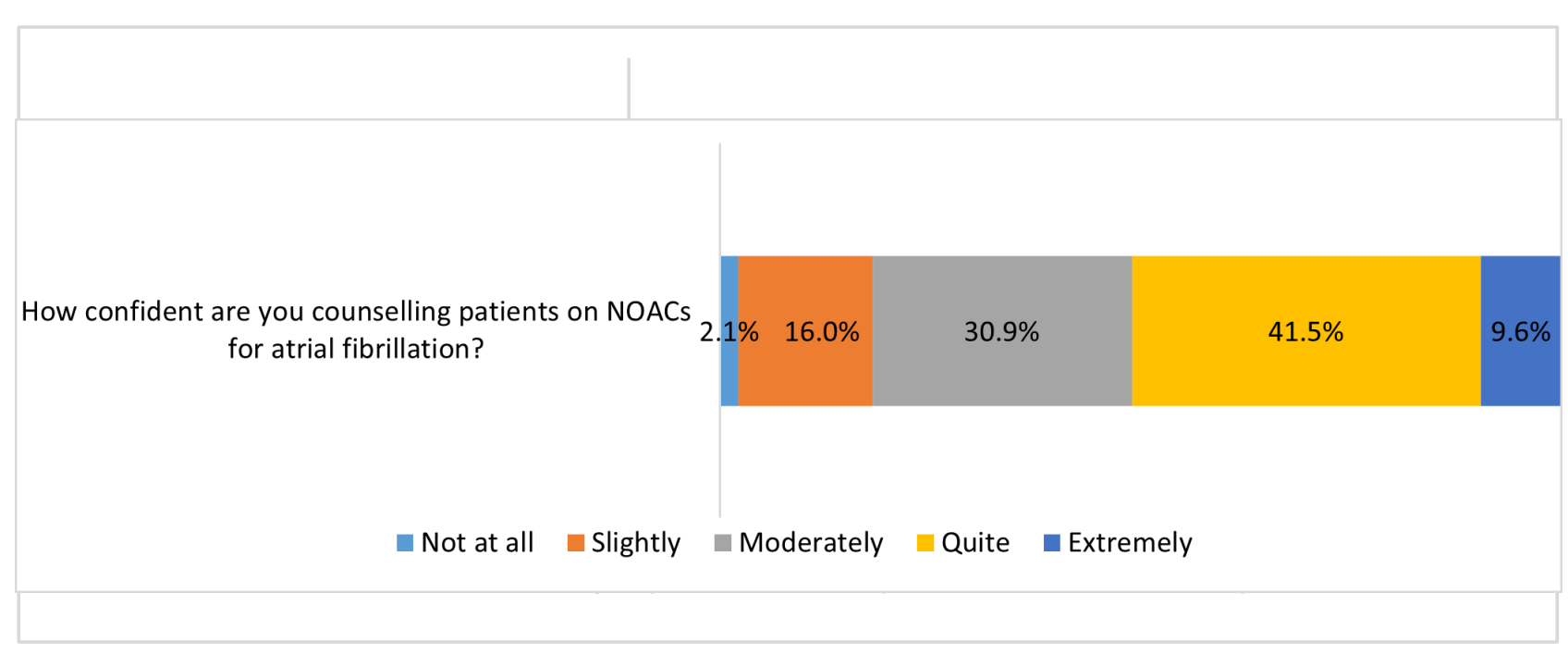

Figure 2: Responses to the Likert question concerning confidence in NOAC counselling

anticoagulant counselling as being essential counselling points (National Institute for Health and Care Excellence, 2014). The questions used five-point Likert scales of 'poor' to 'excellent' and they did not directly test knowledge but reported a respondent's perception of their ability. The questionnaire also included 5-point Likert scales on the implications of and consequences for using the application; there was one ranking question that asked respondents to rank five options from the most to least relevant. Across the questionnaire, the Likert questions were based on previous evaluations of VPs from the literature, the scales used verbal descriptors that have previously been used in VP evaluations (Douglass et al., 2013; Taglieri et al., 2017). A final, free-text, question asked the respondent their perspectives on the consequences of using the VP for patient care.

The questionnaire data were analysed using a mixture of descriptive and inferential statistics. Likert data concerning pre-VP practice and confidence on NOAC counselling, and questions concerning the implications for using the VP were analysed descriptively via medians and interquartile ranges (IQR). Pre-and post-tests of self-perceived counselling ability were grouped together into pre- and post-VP scores and the average percentage change was calculated (Boone \& Boone, 2012). The grouped data underwent statistical analysis using paired t-tests to establish whether there was a significant difference between the two sets of scores pre to post-VP use; T-tests have previously been used in similar VP evaluations (AlDahir et al., 2014; Battaglia et al., 2012). The last sections of analysis included the responses to the ranking question with five outcomes being analysed using a Friedman Test that identified whether there was a statistical significance between the responses (Field, 2013). The free-text question on consequences for patient care was analysed via thematic analysis to identify key perspectives.

\section{Results}

Questionnaire responses were split across those from the hospital sector ( $n=61,64.9 \%$ ) and those from the community sector ( $n=26,27.7 \%, n=7$ reported other sectors of practice or mixed roles) and there was a range in the demographics of the respondents (total respondents $n$ = 94; see Supplementary Material).

Initially respondents were asked how confident they were about conducting NOAC counselling using a scale of 'not at all' to 'extremely' and $72.4 \%$ of responses were for 'moderately' and 'quite' confident (median 'quite', IQR = 1) (Figure 2).

The respondents were also asked about their interest in learning more about NOACs and atrial fibrillation (AF) (from 'not at all' to 'extremely'). Responses were more consistent for this question, with the majority of answers (90.5\%) corresponding to 'quite' and 'extremely' (median 'quite', IQR =1). The same was true for the question about the potential usefulness of such training, where the median was 'extremely' and $94.7 \%$ of responses were for 'quite' and 'extremely' (IQR = 1) (Figure 3).

Respondents were asked to rate their perceived ability to counsel a patient on given sub-areas of NOAC counselling, these were asked before and after use of the VP. Likert data were grouped in to pre- and post-VP scores and underwent a t-test calculation. On average, respondents' 


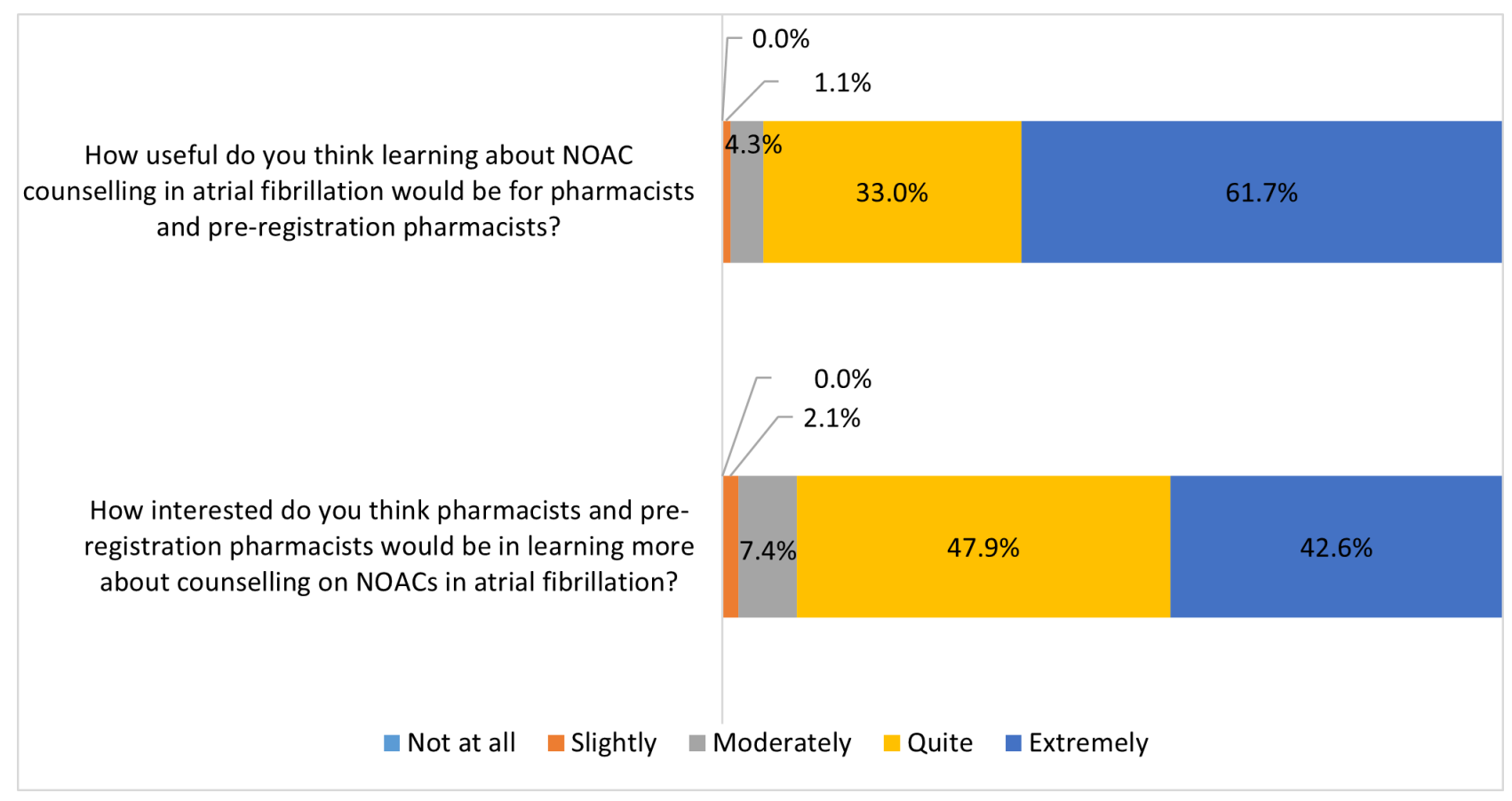

Figure 3: Responses to two Likert questions concerning the usefulness of further learning on NOACs and the interest in further learning on NOACs

scores increased after VP use, with an average increase of $10.2 \%$ (percentage change in ability pre-post VP use ranged from $-30.3 \%-39.6 \%$ ) (Table I), this was statistically significant $(p<0.001)$. Of the 94 respondents, 71 (75.5\%) demonstrated an increase in self-reported ability, 17 (18.1\%) had no change and six (6.4\%) had a decrease.

Table I: Analysis of the significance of the average change in pre- to post-VP self-reported ability using a paired $t$ -

\begin{tabular}{lllll}
\hline test & Mean & $95 \% \mathrm{Cl}^{*}$ & $\begin{array}{l}\text { Standard } \\
\text { deviation }\end{array}$ & Significance (2-tailed) \\
\hline Pre-test & 42.27 & $40.8-43.7$ & 7.13 & - \\
& & & & \\
$\begin{array}{l}\text { Post-test } \\
\begin{array}{l}\text { Difference pre- } \\
\text { to post-VP }\end{array}\end{array}$ & 4.89 & $46.0-48.3$ & 5.59 & - \\
\hline
\end{tabular}

${ }^{*} \mathrm{Cl}$ : Confidence Interval

Further questions asked whether respondents thought that the VP had changed their knowledge, their confidence, and whether this would result in a change in their practice (Figure 4). These questions used 5-point Likert scales of 'strongly disagree' to 'strongly agree'. The spread of the responses for the three questions were similar and the median scores were identical (median 'quite', IQR = 0 for knowledge, IQR = 1 for confidence and change of practice). The question regarding confidence had the lowest proportion of 'agree' and 'strongly agree' responses (70.2\%) although this was close to the scores of the other two questions $(72.3 \%$ knowledge, $75.5 \%$ change of practice). It is unclear whether there was a greater perceived effect of one of these outcomes over the other. Where there were responses that disagreed with this, they tended to be across all three questions.

After using the VP, the respondents ranked five outcomes to indicate the way in which they perceived that it had impacted on their learning and development (1 greatest impact to 5 smallest impact) (Figure 5). 'Change of knowledge' and 'change of confidence' received scores corresponding to the largest impact (average ranking 3.3 and 3.6 respectively) followed by a 'development in generic consultation skills' and 'contribution of CPD' (continuing professional development) (3.1 and 3.0 respectively). Finally, the outcome with the least impact concerned a change of knowledge around NOACs more generally in other indications outside of atrial fibrillation $(A F, A F$ is the indication of the patient within the VP simulation) (2.0).

The significance of this result was assessed via a Friedman Test (Table II), which indicated that the VP outcomes posed to the respondents were ranked statistically differently, $\chi^{2}(4)=52.175,(p=0.000)$. It is unclear exactly where this significance lies. On an individual level, the majority of respondents ranked the 


\section{Implications of VP use}

As a result of using the virtual patient programme, in the future I will change my practice when counselling patients on NOACs for atrial fibrillation

$12.8 \% \quad 12.8 \%$

The virtual patient programme increased my confidence regarding counselling on NOACs for atrial fibrillation

The virtual patient programme increased my knowledge regarding counselling on NOACs for atrial fibrillation.

$8.5 \% \quad 12.8 \%$
(

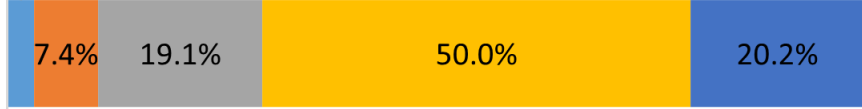

$3.2 \%$

$52.1 \%$

$23.4 \%$

Figure 4: Implications of VP use across three Likert questions

\section{Please rank the following outcomes in terms of the way the virtual patient programme impacted your learning and development}

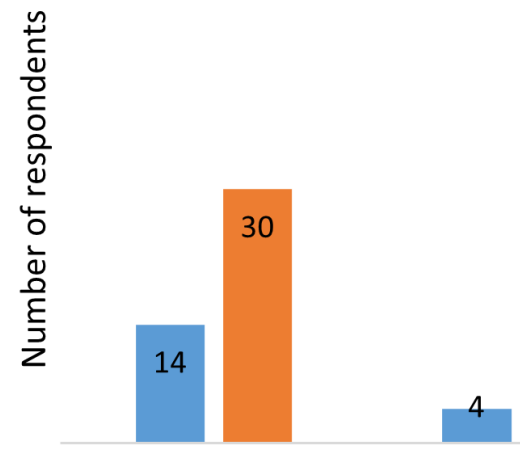

Change of knowledge Change of confidence regarding counselling counselling on NOACs on NOACs in atrial in atrial fibrillation fibrillation

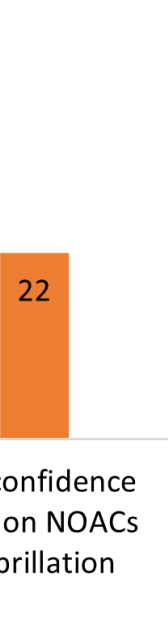

1 - smallest impact

10
51

14

Contribution to continuing professional development 
Table II: Results of a Freidman test for analysing the results of a ranking question concerning the purposes of the VP

\begin{tabular}{lc}
\hline & Friedman Test \\
\hline$N$ & 94 \\
Chi-Square & 52.175 \\
Asymp. Sig. & 0.000 \\
\hline
\end{tabular}

outcomes in roughly the same order as the average rankings, but some individuals appeared to perceive the purpose of the VP as completely different. For example, some individual respondents reported a 'contribution to CPD' or 'contribution to generic consultation skills' as having the greatest impact with 'knowledge and confidence changes' ranked lower down. 'Contribution to CPD' and 'development of generic consultation skills' both received a similar number of rankings for both the largest and smallest impacts, this further highlighted that different users appeared to perceive the purpose of the VP differently to each other.

Finally, respondents were asked what they perceived the potential impact that the use of the VP would have on patient care. Responses included benefits for pharmacist's counselling skills, both, through knowledge acquisition and the opportunity to practice.

'the virtual patient programme could help improve pharmacist patient counselling skills thus improving patient experience. It will also enable pharmacists to practice counselling patients enabling them to feel more confident in counselling patients and be more familiar with the process'

The consequences of this learning for patient care was also related to patient safety and compliance:

'If all new medicines especially high-risk medicines like DOACs [direct oral anticoagulants] and VKAs [vitamin $K$ antagonists] were explained in this manner it would have a hugely positive effect on patient adherence and safety'

A number of respondents also commented on the usefulness of practical experience for conducting this type of counselling. '[the VP] could play a significant role in improving counselling skills and improve skills for a particular subject without practicing on real patients. Improve confidence to talk to patients on a subject'

A number of respondents also directly related the use of the VP to either pre-registration training or as part of CPD.

\section{Discussion}

Most users reported seeing a personal benefit to VP use via an improvement in their perceived ability, this was demonstrated via the measured average increase in selfreported counselling ability pre- to post-VP, although some results were deviant of this. This measured improvement could be due to a number of possible outcomes including both knowledge and confidence changes as well as more generic outcomes regarding counselling ability. This includes the possibility that multiple outcomes can be developed at any one time from using the VP. It is not clear whether the VP was more likely to have an effect on any single particular outcome.

The findings are significant as this is the first study to explicitly explore the use of this type of animated VP by registered pharmacists. Furthermore, the findings demonstrate the concept of a multi-purpose VP where outcomes for individuals may vary or be a blend of multiple. Zigmont and authors discussed that simulation can help individuals to set personal learning goals but this has not yet been explored relative to VPs specifically as a sub-type of simulation technology (Zigmont, Kappus, \& Sudikoff, 2011). Equally, setting goals has previously not been translated to personal outcomes or implications for use and so these findings are fundamental to understanding how VPs could be used by a group of healthcare professionals where outcomes may not be standardised. Furthermore, the respondents recognised the potential impact of the VP to increase counselling standards and consequently patient care. This is encouraging as VP use has scarcely been linked to outcomes in practice, with no studies appearing to focus on the use of VP by healthcare professionals relative to the service that patients receive.

The majority of respondents identified VP use for NOACspecific knowledge and confidence outcomes over other options, although there was very little between the two as they appear to be interlinked. For other users the important outcomes were somewhat reversed relating to 
generic counselling and CPD. This could go some way to explaining those participants where there was no change in self-perceived ability to conduct NOAC counselling preto post-VP. As for some users the benefits appeared to be related to more general outcomes to using the VP such as development of consultation skills and less so concerning NOAC counselling specifically. Whilst this question contributed to establishing the overall benefit and purpose to using the VP it also clear that in some cases the purpose or outcome of using the VP was individualised. Significantly no users were overwhelmingly negative about their experience of using the VP and the majority indicated that their learning from using the VP would still change their practice.

Some respondents demonstrated a decrease in selfreported ability pre- to post-VP but largely these users were still positive about using the VP. One possible explanation is that the VP helped users to identify areas where they needed improvement hence their ability postVP was scored lower than pre-VP as their ability was not as good as they had initially perceived (pre-VP). This concept was commented on in the work of Botezatu and authors who identified that VPs in medical students had a regulatory effect and helped to plan learning (Botezatu et al., 2010). Similarly in the study by Forsberg and authors, nursing students reported feeling uncertain as the use of a VP had exposed knowledge gaps (Forsberg et al., 2016). Within Pharmacy the only study to recognise this phenomena was Zary and authors., who suggested that their VP could identify knowledge gaps and motivate knowledge acquisition, although this was not measured (Zary et al., 2006). It could be that for some users this has occurred, and the VP has helped identify previously unknown deficits in knowledge or practice.

This study represents a single VP application and its resulting evaluation, currently VPs are highly individualised applications that each require their own evaluation. The transferability of the findings depends on the similarly of technologies and the educational resources. The description and detail provided here is intended to facilitate assessments of such similarities.

\section{Conclusion}

There was an average improvement in pharmacist's and pre-registration pharmacist's self-perceived counselling ability and the VP appeared to be beneficial to the majority of users although this was not always via an ability change but may have included regulatory effects and non-subject specific benefits. Users were positive about the implications of using the VP with a number of learning outcomes identified, although these varied between individuals. It is possible that there are a number of educational benefits for users of the VP with no single overarching purpose applicable to all.

\section{Funding}

The VP was commissioned and paid for by, Bayer AG. They had no involvement in the design or conduct of the research nor in the drafting of this manuscript. There are no competing interests concerning this study. This research did not receive any specific grant from funding agencies in the public, commercial, or not-for-profit sectors. Henry Gentle, the virtual patient character is not an actual patient. Any resemblance to real person living or deceased is a coincidence.

\section{References}

Al-Dahir, S., Bryant, K., Kennedy, K.B., \& Robinson, D.S. (2014). Online virtual-patient cases versus traditional problem-based learning in advanced pharmacy practice experiences. American Journal of Pharmaceutical Education, 78(4), 1-8. https://doi.org/ 10.5688/ajpe78476

Battaglia, J.N., Kieser, M.A., Bruskiewitz, R.H., Pitterle, M.E., \& Thorpe, J.M. (2012). An online virtual-patient program to teach pharmacists and pharmacy students how to provide diabetesspecific medication therapy management. American Journal of Pharmaceutical Education, 76(7), 131. https://doi.org/10.5688/ ajpe767131

Bindoff, I., Ling, T., Bereznicki, L., Westbury, J., Chalmers, L., Peterson, G., \& Ollington, R. (2014). A computer simulation of community pharmacy practice for educational use. American Journal of Pharmaceutical Education, 78(9), 1-8. https://doi.org/ 10.5688/ajpe789168

Boone, H.N., \& Boone, D.A. (2012). Analyzing Likert Data LikertType Versus Likert Scales (online). Journal of Extension, 50(2). Available from: https://archives.joe.org/joe/2012april/pdf/ JOE_v50_2tt2.pdf

Botezatu, M., Hult, H., \& Fors, U.G. (2010). Virtual patient simulation: what do students make of it? A focus group study. BMC Medical Education, 10(1), 91. https://doi.org/ 10.1186/1472-6920-10-91 
Bracegirdle, L., \& Chapman, S. (2010). Programmable Patients: Simulation of Consultation Skills in a Virtual Environment. BioAlgorithms and Med-Systems, 6(11), 111-115.

Cavaco, A. M., \& Madeira, F. (2012). European pharmacy students' experience with virtual patient technology. American Journal of Pharmaceutical Education, 76(6), 1-7. https://doi.org/ 10.5688/ajpe766106

Douglass, M., Casale, J., Skirvin., A., \& DiVall, M. (2013). A virtual patient software program to improve pharmacy student learning in a comprehensive disease management course. American Journal of Pharmaceutical Education, 77(8), 172. https://doi.org/ 10.5688/ajpe778172

Duff, E., Miller, L., \& Bruce, J. (2016). Online Virtual Simulation and Diagnostic Reasoning: A Scoping Review. Clinical Simulation in Nursing, 12(9), 377-384. https://doi.org/10.1016/j.ecns. 2016.04.001

Field, A. (2013). Discovering Statistics Using IBM SPSS Statistics (Fourth Ed.). London: SAGE.

Forsberg, E., Ziegert, K., Hult, H., \& Fors, U. (2016). Assessing progression of clinical reasoning through virtual patients: An exploratory study. Nurse Education in Practice, 16(1), 97-103. https://doi.org/10.1016/j.nepr.2015.09.006

Kane-Gill, S., \& Smithburger, P. (2011). Transitioning Knowledge Gained From Simulation to Pharmacy Practice. American Journal of Pharmaceutical Education, 75(10), 210. https://doi.org/ 10.5688/ajpe7510210

Menendez, E., Balisa-Rocha, B., Jabbur-Lopes, M., Costa, W., Nascimento, J., Dosea, M., Silva, L., \& Junior, D.L. (2015). Using a virtual patient system for the teaching of pharmaceutical care. International Journal of Medical Informatics, 84(9), 640-646. https://doi.org/10.1016/j.ijmedinf.2015.05.015

National Institute for Health and Care Excellence. (2014). Atrial fibrillation: management (online). Available from: https:// www.nice.org.uk/guidance/cg180

Pereira, D., \& Cavaco, A. (2014). Exploring computer simulation to assess counseling skills amongst pharmacy undergraduates. Indian Journal of Pharmaceutical Education and Research, 48(1), 17-26. https://doi.org/10.5530/ijper.48.1.4

Richardson, C.L., Chapman, S., \& White, S. (2019). Virtual patient educational programme to teach counselling to clinical pharmacists: Development and proof of concept. BMJ Simulation and Technology Enhanced Learning, 5(3). https:// doi.org/10.1136/bmjstel-2018-000352

Richardson, C.L., White, S., \& Chapman, S. (2019). Virtual patient technology to educate pharmacists and pharmacy students on patient communication: a systematic review. BMJ Simulation and Technology Enhanced Learning. https://doi.org/10.1136/ bmjstel-2019-000514
Smith, M., \& Benedict, N. (2015). Effectiveness of educational technology to improve patient care in pharmacy curricula. American Journal of Pharmaceutical Education, 79(1), 15. https://doi.org/10.5688/ajpe79115

Smith, M., Mohammad, R., \& Benedict, N. (2014). Use of virtual patients in an advanced therapeutics pharmacy course to promote active, patient-centered learning. American Journal of Pharmaceutical Education, 78(6), 125. https://doi.org/10.5688/ ajpe786125

Stevens, A., Hernandez, J., Johnsen, K., Dickerson, R., Raij, A., Harrison, C., DiPietro, M., Allen, B., Ferdig, R., foti, S., Jackson, J., Shin, M., Cendan, J., Watson, R., Duerson, M., Lok, B., Cohen, M., Wagner, P., \& Lind, D.S. (2006). The use of virtual patients to teach medical students history taking and communication skills. American Journal of Surgery, 191(6), 806-811. https://doi.org/ 10.1016/j.amjsurg.2006.03.002

Taglieri, C.A., Crosby, S.J., Zimmerman, K., Schneider, T., \& Patel, D.K. (2017). Evaluation of the use of a virtual patient on student competence and confidence in performing simulated clinic visits. American Journal of Pharmaceutical Education, 81(5). https:// doi.org/10.5688/ajpe81587

Thompson, J., White, S., \& Chapman, S. (2016). A qualitative study of the perspectives of pre-registration pharmacists on the use of virtual patients as a novel educational tool. International Journal of Pharmacy Practice, 24(S3), 34. https://doi.org/ 10.1111/ijpp.12289/full

Thompson, J., White, S., \& Chapman, S. (2017). Trainee perceptions on the usefulness of Virtual Patients and other support during the pre-registration year. International Journal of Pharmacy Practice, 25(S1), 33-34. https://doi.org/10.1111/ijpp. 12370

Zary, N., Johnson, G., Boberg, J., \& Fors, U.G.H. (2006). Development, implementation and pilot evaluation of a Webbased Virtual Patient Case Simulation environment - Web-SP. BMC Medical Education, 6, 1-17. https://doi.org/ 10.1186/1472-6920-6-10

Zigmont, J.J., Kappus, L.J., \& Sudikoff, S.N. (2011). Theoretical Foundations of Learning Through Simulation. Seminars in Perinatology, 35(2), 47-51. https://doi.org/10.1053/j.semperi. 2011.01.002

Zlotos, L., Power, A., Hill, D., \& Chapman, P. (2016). A scenariobased virtual patient program to support substance misuse education. American Journal of Pharmaceutical Education, 80(3), 48. https://doi.org/10.5688/ajpe80348 